\title{
Estimates of solutions to nonlinear evolution equations
}

\author{
A. G. Ramm \\ Mathematics Department, Kansas State University, \\ Manhattan, KS 66506-2602, USA
}

\begin{abstract}
Consider the equation

$$
u^{\prime}(t)=A(t, u(t)), \quad u(0)=u_{0} ; \quad u^{\prime}:=\frac{d u}{d t}
$$

Under some assumptions on the nonlinear operator $A(t, u)$ it is proved that problem (1) has a unique global solution and this solution satisfies the following estimate

$$
\|u(t)\|<\mu(t)^{-1} \quad \forall t \in \mathbb{R}_{+}=[0, \infty) .
$$

Here $\mu(t)>0, \mu \in C^{1}\left(\mathbb{R}_{+}\right)$, is a suitable function and the norm $\|u\|$ is the norm in a Banach space $X$ with the property $\|u(t)\|^{\prime} \leq\left\|u^{\prime}(t)\right\|$.
\end{abstract}

Mathematics Subject Classification: MSC 2010, 47J05; 47J35; 58D25

Keywords: nonlinear evolution equations

\section{Introduction}

Let

$$
u^{\prime}=A(t, u(t)), \quad u(0)=u_{0} ; \quad u^{\prime}:=\frac{d u}{d t},
$$

where $t \in \mathbb{R}_{+}=[0, \infty), A(t, u)$ is a locally continuous map from $\mathbb{R}_{+} \times X$ into $X$, where $X$ is a Banach space of functions with the norm $\|\cdot\|$, such that $\|u(t)\|^{\prime} \leq\left\|u^{\prime}(t)\right\|$ if $u(t)$ is continuously differentiable with respect to $t$. If $u(t) \in X$ is a function then $|u(t)|$ and $\|u(t)\|$ make sense. We assume that if $|u| \leq|v|$ then $\|u\| \leq\|v\|$. For the spaces of continuous functions and $L^{p}$ spaces this assumption holds.

We assume that

$$
\|A(t, u)-A(t, v)\| \leq k\|u-v\|,
$$

Corresponding author: Email: ramm@math.ksu.edu

ISSN: 2347-1921

Volume:14 Issue: 2

DOI: 10.24297/jam.v14i2.7445

Journal: Journal of Advances in Mathematics 
where $k>0$ is a constant which may depend on $R,\|u\| \leq R,\|v\| \leq R$, and on $T, t \in[0, T]$.

If $A(t, u)$ is a function with values in $\mathbb{R}$ and $\|A(t, u)\|=|A(t, u)|$, then (1) is a nonlinear ordinary differential equation and condition (2) guarantees local existence and uniqueness of its solution on an interval $[0, T]$ where $T$ is a sufficiently small number. If $T=\infty$ then the solution $u(t)$ is called global.

The map $A(t, u)$ may be of the form

$$
A(t, u)=\int_{0}^{t} a(t, s, u(s)) d s
$$

where $a(t, s, u)$ is a locally continuous function on $\mathbb{R}_{+} \times R_{+} \times X$, locally Lipschitz with respect to $u$.

The following assumptions will be valid throughout this paper:

There exists a $C^{1}\left(\mathbb{R}_{+}\right)$function $\mu(t)>0$ such that

$$
\left\|A\left(t, \frac{w}{\mu(t)}\right)\right\| \leq\left(\frac{1}{\mu(t)}\right)^{\prime},
$$

where $\|w\|=1, w \in X$ is an arbitrary element,

$$
\|A(t, u)\| \leq\|A(t, v)\| \quad \text { if } \quad|u| \leq|v|
$$

and

$$
\|u(0)\|<\frac{1}{\mu(0)}
$$

Theorem 1. Under the above assumptions the solution to (1) exists globally, is unique, and satisfies the following estimate:

$$
\|u(t)\|<\frac{1}{\mu(t)}, \quad \forall t \in \mathbb{R}_{+} .
$$

Remark 1. Some conditions on $A(t, u)$ of the type (4)- (6) are necessary for the global existence of the solution.

Consider the following example: $u^{\prime}=u^{2}, u(0)=1$. This problem is equivalent to the equation $u=1+\int_{0}^{t} u^{2}(s) d s$. The solution to this problem is $u(t)=(1-t)^{-1}$, so it tends to $\infty$ as $t \rightarrow 1$. The solution is smooth on $[0, \lambda]$, where $0<\lambda<1$ is arbitrary.

\section{Proofs}

The proof of Theorem 1 consists of several parts. We start with the part dealing with the inequality

$$
\|u(t)\|^{\prime} \leq\left\|u^{\prime}(t)\right\|
$$


We assume throughout that $u(t)$ is continuously differentiable with respect to $t$.

2.1. Inequality (8) holds if $X=H$, where $H$ is a Hilbert space. The inner product in $H$ is denoted as usual $(u, v)$. A simple proof of (8) goes as follows. Start with the inequality

$$
\frac{\|u(t+h)\|-\|u(t)\|}{h} \leq\left\|\frac{u(t+h)-u(t)}{h}\right\|
$$

and let $h \rightarrow 0$. The result is (8). Indeed, the limit of the right side does exist and is equal to $\left\|u^{\prime}(t)\right\|$. To calculate the limit of the left side in (9) consider the identity

$$
\begin{gathered}
h^{-1}(\|u(t+h)\|-\|u(t)\|)(\|u(t+h)\|+\|u(t)\|)= \\
h^{-1}(u(t+h)-u(t), u(t+h))+h^{-1}(u(t), u(t+h)-u(t)) .
\end{gathered}
$$

Clearly, the limit of the right side exists and is equal to $2 \operatorname{Re}\left(u^{\prime}(t), u(t)\right)$. One has $\lim _{h \rightarrow 0}(\|u(t+h)\|+\|u(t)\|)=2\|u(t)\|$. Assuming that $\|u(t)\|>0$ one concludes that

$$
\|u(t)\|^{\prime}=\lim _{h \rightarrow 0} h^{-1}(\|u(t+h)\|-\|u(t)\|)=\operatorname{Re}\left(u^{\prime}(t), u(t)\right) /\|u(t)\| \leq\left\|u^{\prime}(t)\right\| .
$$

If $\|u(t)\|=0$, then $\|u(t)\|^{\prime}=\lim _{h \rightarrow 0} h^{-1}\|u(t+h)\|$. One has $\|u(t+h)\|^{2}=$ $(u(t+h), u(t+h))=h^{2}\left\|u^{\prime}(t)\right\|^{2}+o\left(h^{2}\right)$. Thus, $\|u(t+h)\|=|h|\left\|u^{\prime}(t)\right\|+o(h)$. Therefore $\|u(t)\|^{\prime}=\lim _{h \rightarrow 0} h^{-1}|h|\left\|u^{\prime}(t)\right\|=\operatorname{sign} h\left\|u^{\prime}(t)\right\| \leq\left\|u^{\prime}(t)\right\|$. Formula (8) is proved for $X=H$.

If $X=\mathbb{R}$ the proof of (8) is left for the reader. One gets $\left.|| u(t)\right|^{\prime}|\leq| u^{\prime}(t) \mid$.

2.2. Let us study problem (1) assuming that $X=\mathbb{R}, w=1$ in (4) and $\|u(t)\|=|u(t)|$. Assumption (2) guarantees local existence and uniqueness of the solution to (1). We want to prove that assumptions (4)-(6) guarantee the global existence of the solution $u(t)$ and estimate (7). If (6) holds, then, by continuity, there exists a small $\delta>0$ such that

$$
\mid u(t)<\frac{1}{\mu(t)}, \quad 0 \leq t \leq \delta .
$$

This and (5) imply

$$
|A(t, u(t))| \leq\left|A\left(t, \frac{1}{\mu(t)}\right)\right|, \quad 0 \leq t \leq \delta .
$$

Take the absolute value of (1), use (7), (11) and (4) to get

$$
|u(t)|^{\prime} \leq|A(t, u(t))| \leq\left|A\left(t, \frac{1}{\mu(t)}\right)\right| \leq\left(\frac{1}{\mu(t)}\right)^{\prime}, \quad 0 \leq t \leq \delta .
$$


Integrating 12 with respect to $t$ one gets

$$
|u(t)|-|u(0)| \leq \frac{1}{\mu(t)}-\frac{1}{\mu(0)}, \quad 0 \leq t \leq \delta .
$$

This and (6) imply (7) for $t \in[0, \delta]$. Define $T$ as follows:

$$
T=\sup \left\{\delta: \mid u(t)<\frac{1}{\mu(t)}, \quad 0 \leq t \leq \delta\right\} .
$$

Let us prove that $T=\infty$.

Assuming the contrary, i.e., $T<\infty$, one uses the local existence of the solution to (1) taking as initial value $u(T)$ and as the interval of the existence of the solution $[T, T+h]$, where $h>0$ is a sufficiently small number. Then inequality (7) holds for $t \in[0, T+h]$. This contradicts to the definition (14) of $T$. So, one gets a contradiction which proves that $T=\infty$ and estimate (7) holds for all $t \in \mathbb{R}_{+}$. Theorem 1 is proved for $X=\mathbb{R}$.

2.3. Consider the nonlinear Volterra equation:

$$
u(t)=\int_{0}^{t} a(t, s, u(s)) d s+f(t) .
$$

Assume that $a(t, s, u)$ and $a_{t}:=\frac{\partial a}{\partial t}$ are continuous functions on $\mathbb{R}_{+} \times \mathbb{R}_{+} \times \mathbb{R}$, locally Lipschitz with respect to $u$. Differentiate (15) with respect to $t$ and get

$$
u^{\prime}=a(t, t, u(t))+\int_{0}^{t} a_{t}(t, s, u(s)) d s+f^{\prime}(t):=A_{1}(t, u(t)) .
$$

Assume that $A_{1}(t, u)$ satisfies conditions (4)-(6) with $w=1$, and $\|u(t)\|=$ $|u(t)|$. Then the argument used in scetion 2.2. proves Theorem 1 with $A_{1}(t, u)$ replacing $A(t, u)$.

Example 1. The aim of this example is to derive sufficient conditions on $a(t, s, u)$ for the assumptions (4)-(6) to hold. Let

$$
\begin{array}{r}
|a(t, s, u)|+\left|a_{t}(t, s, u)\right| \leq c e^{-b(t+s)}\left(1+|u|^{2 m}\right), \quad m>1 \\
|f(t)|+\left|f^{\prime}(t)\right| \leq c e^{-b t}
\end{array}
$$

where $c, b>0$ are constants. We assume that $a$ and $a_{t}$ are Lipschitz functions with respect to $u$. Assume that

$$
\begin{gathered}
|a(t, t,|u|)| \leq|a(t, t,|v|)| \quad \text { if } \quad|v| \geq|u|, \\
\left|a_{t}(t, t,|u|)\right| \leq\left|a_{t}(t, t,|v|)\right| \quad \text { if } \quad|v| \geq|u| .
\end{gathered}
$$

Let

$$
\mu(t)=c_{0} e^{-a t}, \quad a>0
$$


Note that $\left(\frac{1}{\mu(t)}\right)^{\prime}=a c_{0}^{-1} e^{a t}$. If (17) holds, then the following two inequalities

$$
\begin{array}{r}
\left|f^{\prime}(t)\right|+\left|a\left(t, t, c_{0}^{-1} e^{a t}\right)\right| \leq c e^{-b t}+c e^{-2 b t}\left(1+c_{0}^{-2 m} e^{2 m a t}\right) \leq \\
0.5 a c_{0}^{-1} e^{a t}=0.5\left(\frac{1}{\mu(t)}\right)^{\prime}, \\
\int_{0}^{t}\left|a_{t}\left(t, s, c_{0}^{-1} e^{a s}\right)\right| d s \leq \int_{0}^{t} c e^{-b(t+s)}\left(1+e^{2 m a s} / c_{0}^{2 m}\right) d s \leq \\
c e^{-b t}\left[\left(1-e^{-b t}\right) / b+\left(1-e^{-(b-2 m a) t}\right) /\left[c_{0}^{2 m}(b-2 m a)\right] .\right.
\end{array}
$$

and conditions (4)-(5) hold provided that

$$
c / b+1 /\left[c_{0}^{2 m}(b-2 m a)\right] \leq a /\left(2 c_{0}\right), \quad b>2 m a,
$$

where $b$ is sufficiently large and $c$ is sufficiently small. If in addition (6) holds, i.e., $c c_{0}<1$, then $u(t)$ exists globally and the estimate $|u(t)|<c_{0}^{-1} e^{a t} \quad \forall t \in \mathbb{R}_{+}$ holds.

2.4. Consider equation (1) in $X$. Assume that conditions (2), (4)- (6) and (8) hold. Then there is a unique local solution to (1) continuous with respect to $t$ in $X$. It follows from (4)-(6) that

$$
\|u(t)\|^{\prime} \leq \| A\left(t, u(t)\|\leq\| A(t, w / \mu(t)) \|<(1 / \mu(t))^{\prime}, \quad 0 \leq t \leq \delta .\right.
$$

Here $\delta>0$ is sufficiently small so that $\|u(t)\|<1 / \mu(t)$ for $0 \leq t \leq \delta$. Integrate (22) on any interval $[0, T]$ on which the solution $u(t)$ exists one gets $\|u(t)\|<$ $1 / \mu(t)$ for $t \in[0, T]$. As in section 2.3 we prove that $T=\infty$. Therefore problem (1) has a unique global solution in $X$ and estimate (7) holds.

Theorem 1 is proved.

The ideas close to the ones used in this paper were developed and used in $[1]-[3]$.

\section{References}

[1] Ramm, A. G., Stability of the solutions to evolution problems, Mathematics, 1, (2013), 46-64.

doi:10.3390/math1020046

Open access Journal:

http://www.mdpi.com/journal/mathematics

[2] Ramm, A. G., Large-time behavior of solutions to evolution equations, Handbook of Applications of Chaos Theory, Chapman and Hall/CRC, 2016, pp. 183-200 (ed. C.Skiadas). 
[3] Ramm, A. G., Hoang, N. S., Dynamical Systems Method and Applications. Theoretical Developments and Numerical Examples. Wiley, Hoboken, 2012. 